\title{
A molecular jigsaw puzzle
}

\author{
Convergent efforts of researchers from different fields aim to control spin transport in molecules and \\ develop nanoscale spintronic devices with improved performance or new functionalities.
}

Ever since the discovery of conducting polymers, organic molecules and salts in the 1960s and 1970s, conducting organic solids and their potential for electronic and photonic functions have attracted interest $\mathrm{t}^{1-3}$. This has been driven by their mechanical flexibility and their large-scale, low-cost production compared with their inorganic counterparts. When devices exploiting the electronic properties of single or few molecules were also proposed, aiming at reaching the ultimate limits of electronic circuit miniaturization ${ }^{4}$, organic and molecular electronics were born.

Successful experimental exploration of spin transport in carbon-based materials occurred in the late 1990s with the pioneering work by Kazuhito Tsukagoshi and colleagues ${ }^{5}$. By contacting a multi-walled carbon nanotube with ferromagnetic electrodes and measuring the magnetoresistance response (a change in resistance depending on the parallel or antiparallel alignment of the electrodes' magnetization), they demonstrated the possibility for a spin-polarized current to be injected and coherently transported through the carbon nanotube. Soon after, Valentin Alek Dediu and collaborators, while working on organic light-emitting diodes, discovered that heterostructures formed by an organic layer sandwiched between ferromagnetic electrodes can show large magnetoresistance, an observation that gave birth to the field of organic spintronics ${ }^{6}$. The longer spin lifetimes of organic materials compared to inorganics propelled this research field; spin injection and transport in other hybrid organic semiconductor heterostructures, as well as the use of these materials as spin-tunnel barriers, were later demonstrated ${ }^{7}$.

In parallel, efforts were made to gain insight into spin transport at the molecular level. Many theoretical works have been devoted to understanding spin injection through single molecules ${ }^{8}$, and spin-polarized scanning tunnelling microscopy has allowed the direct exploration of it in molecules absorbed into surfaces 9 . Nevertheless, the realization of devices - such as magnetic single-molecular junctions - for a straightforward quantitative comparison between predictions and measurements is still missing. Beyond that, the ultimate goal of molecular spintronics is the realization of devices with new or improved functionalities directly relying on molecular properties and their miniaturization down to the molecular level. For example, molecular magnets in which properties of bulk magnetic materials and molecular quantum effects coexist - have received a lot of attention, with a view to detect and manipulate molecular spin for high-density information storage and the realization of spin qbits for quantum computing ${ }^{10}$.

Organic and molecular spintronics have mainly followed independent courses in the past few decades, but recently it seems that the two paths are converging. For instance, studies at the molecular level are now able to provide insight into the transport properties of organic spintronic devices. This is particularly true in material systems whose behaviour is dominated by interfacial effects.

In 2010, Clément Barraud and co-workers found that the interface between organic and inorganic materials can behave as a spinactive layer that can dominate spin-transport properties of organic spintronic devices, leading to the concept of the spinterface ${ }^{11,12}$. The interface between organic and inorganic materials is a versatile playground to study emerging spin properties arising from molecular absorption and hybridization on substrates, charge transfer and even chemical reactions. For instance, these interfaces have been found to determine the magnetic properties of both the inorganic and organic component of some heterostructures, and to be able to stabilize molecular magnetism at high temperature ${ }^{13}$. Moreover, charge transfer at the molecular interface of non-magnetic heterostructures can even lead to roomtemperature magnetism ${ }^{14}$. On page 507 of this issue, Mirko Cinchetti, Valentin Dediu and Luis Hueso review our current understanding of molecular spinterfaces and discuss how the interfacial spin effects could be used to develop electronic devices. In particular, they suggest device architectures that would take advantage of the chemical functionality of and molecular responsivity to electrical and optical stimuli.

Molecular spintronics is a truly multidisciplinary research field made up of different jigsaw pieces that are beginning to be put togeher. The European Conference in Molecular Spintronics (http://www.ecmols.eu/), held in Bologna in

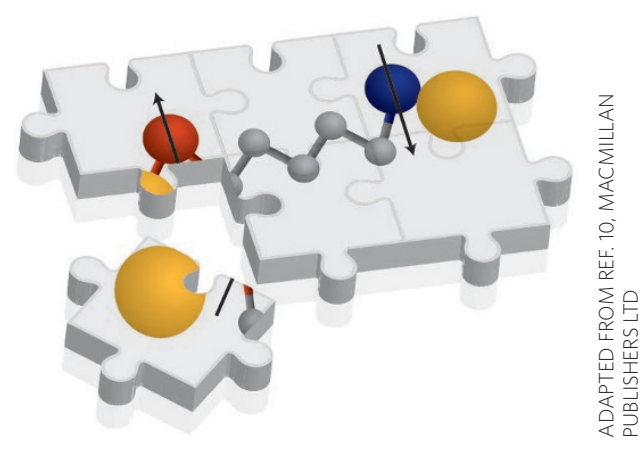

November 2016, aimed to bring together the expertise of materials scientists, physicists and chemists working on organic spintronics, molecular magnetism and molecular quantum technologies. On page 505 Andrea Cornia and Pierre Seneor give an overview of the main ideas put forward during the conference. The topics covered give a clear idea of the interdisciplinary nature of molecular spintronics: molecular-based spin devices including single-molecule spintronics, processing technologies, manipulation of molecular spins through external stimuli, molecular magnetism, spin-orbit induced effects, and the realization and manipulation of molecular qubits for quantum technologies.

Aligning the activities of groups with not only different backgrounds, but also different approaches, interests and perspectives is always challenging. However, unknotting the full potential of molecules for spintronics promises many scientific and technological rewards. It is certainly a puzzle worth solving.

References

1. McNeill, R., Siudak, R., Wardlaw, J. H. \& Weiss, D. E. Aust. J. Chem. 16, 1056-1075 (1963).

2. Mann, B. \& Kuhn, H. J. Appl. Phys. 42, 4398-4405 (1971).

3. Shirakawa, H., Louis, E. J., MacDiarmid, A. G., Chiang, C. K. \& Heeger, A. J. J. Chem. Soc. Chem. Commun. 578-580 (1977).

4. Aviram, A. \& Ratner, M. A. Chem. Phys. Lett. 29, 277-283 (1974).

5. Tsukagoshil, K., Alphenaar, B. W. \& Ago, H. Nature 401, 572-574 (1999).

6. Dediu, V., Mirgoa, M., Matacotta, F. C., Taliani, C. \& Barbanera, S. Solid State Commun. 122, 181-184 (2002)

7. Dediu, V. A., Hueso, L. E., Bergenti, I. \& Taliani, C. Nat. Mater. 8, 707-716 (2009).

8. Sanvito, S. \& Rocha, A. R. J. Comput. Theor. Nanosci. 3, 624-642 (2006).

9. Schmaus, S. et al. Nat. Nanotech. 6, 185-189 (2011).

10. Bogani, L. \& Wernsdorfer, W. Nat. Mater. 7, 179-186 (2008).

11. Barraud, C. et al. Nat. Phys. 6, 615-620, (2010).

12. Sanvito, S. Nat. Phys. 6, 562-564 (2010).

13. Gruber, M. et al. Nat. Mater. 14, 981-984 (2015).

14. Ma’Mari, F. M. et al. Nature 524, 69-73 (2015). 